\title{
Baseline serum folate level may be a predictive factor of weight loss in a morbid-obesity-management programme
}

\author{
J. J. Gorgojo Martínez*, F. Almodóvar Ruiz and S. Donnay Candil \\ Unit of Endocrinology and Nutrition, Fundación Hospital Alcorcón, C/ Budapest 1, 28922 Alcorcón (Madrid), Spain \\ (Received 9 January 2006 - Revised 6 June 2006 - Accepted 6 June 2006)
}

\begin{abstract}
Prompt identification of responders to non-surgical therapy is of utmost importance in attempting medical treatment in patients with clinically severe obesity before indication of bariatric surgery. The objectives of the present study were to assess the outcome at 1 year of morbidly obese patients undergoing a weight-loss medical programme and to detect baseline predictors of a loss $\geq 10 \%$ of initial weight at the end of the follow-up. A longitudinal, prospective study of a cohort of morbidly obese patients ( $n$ 182; females $78 \%$; age 40.5 (SD 11.5 ) years; BMI $45.4(\mathrm{SD} 6 \cdot 0) \mathrm{kg} / \mathrm{m}^{2}$ ) enrolled in a 1-year obesity-management programme based on lifestyle changes and pharmacological therapy. Significant laboratory and clinical variables were included in a binary logistic regression model in order to identify baseline independent factors for the prediction of a successful outcome in the programme. At 12 months of follow-up, twenty-one subjects (11.5\% of the initial cohort) had lost $\geq 10 \%$ of baseline weight. A high serum folic acid level was the only independent predictor of weight loss at 1 year. A rise of $1 \mathrm{ng} / \mathrm{ml} \mathrm{in}$ serum folate increased the chance of success by $28 \%$ (adjusted odds ratio $1.28 ; 95 \%$ CI 1.04, 1.58). We concluded that a medical-management programme of morbid obesity obtained limited results at 1 year, in agreement with other intervention studies. Serum folate may be useful as a pre-treatment predictor of response to a medical-management programme in patients with morbid obesity. Patients with low basal serum folate levels probably should be urged to change unhealthy eating patterns.
\end{abstract}

Morbid obesity: Weight loss: Therapy: Folic acid

Obesity is a major public health problem in developed countries. The prevalence of obesity among the American adult population in the period $1999-2000$ was $30.5 \%$, compared with $22.9 \%$ in the National Health and Nutrition Examination Survey (NHANES) III programme conducted between 1988 and 1994 (Flegal et al. 2002). Morbid or severe obesity increased from 2.9 to $4.7 \%$ during the same period, reaching an extremely high prevalence in some ethnic groups (about $15 \%$ in non-Hispanic black women). In Spain, obesity affects an important percentage of the adult population, although not in the epidemic proportions observed in the USA. The Spanish Society for the Study of Obesity (Sociedad Española para el Estudio de la Obesidad; SEEDO) 2000 study showed a prevalence of obesity and morbid obesity of 14.5 and $0.5 \%$ respectively among the Spanish population aged between 25 and 60 years (Aranceta et al. 2003).

The life expectancy of patients with severe obesity is reduced by $10-15$ years and there is a 12 -fold excess mortality risk in morbidly obese men aged between 25 and 34 years and a 6-fold excess mortality in men between 35 and 44 years old compared with the general population (Drenick et al. 1980). Although a $10 \%$ weight loss is associated with improvements in most obesity-related co-morbidities (National Institutes of Health, 2000), several medical therapies have been shown unsuccessful in most patients with severe obesity, with bariatric surgery emerging as the primary treatment for this pathology (MacDonald, 2003; Sjöström et al. 2004). However, a selected group of patients can respond to a lifestyle modification and/or drug therapy, and in others bariatric surgery is contraindicated because of advanced age or severe psychiatric or medical pathologies. Prompt identification of responders to non-surgical therapy is of utmost importance to reinforce the medical treatment in this subgroup and not delay bariatric surgery in the rest of the patients.

Among biological variables, initial body weight and RMR are both positively related to weight loss, but behavioural predictors have proved to be less consistent (National Institutes of Health, 2000; Teixeira et al. 2004). Early weight loss is a useful clinical indicator of response to pharmacological treatment. A recent study showed that a weight loss $\geq 5 \%$ at 12 weeks was a strong predictive factor of a weight loss $\geq 10 \%$ at 2 years in orlistat-treated obese patients (Rissanen et al. 2003). Nevertheless, no prognostic biochemical markers of successful weight-loss outcome have been defined in morbid obesity. One epidemiological study in non-obese male health professionals showed that high serum folate and low serum insulin and homocysteine levels seem to be biomarkers of a healthy dietary pattern characterised by higher intakes of fruit, vegetables, whole grains and poultry (Fung et al. 2001). Theoretically, these biochemical parameters could be used as surrogate markers of a balanced, low-fat 
diet at the beginning of the therapy. If a markedly obese patient already follows a nutritionally adequate diet before the medical intervention, the likelihood of a successful outcome in an obesity-management programme probably will be higher. Serum folate may be a simple and cheap biomarker of healthy diet and a good predictor of response to the obesity therapy.

Published studies of medical therapies include few reports or indications of efficacy in individuals with clinically severe obesity. In 2000, a morbid-obesity-management programme, reflecting a real-life clinical setting and based in lifestyle changes and pharmacological therapy before surgical indication, was started at our institution. We proposed to evaluate the results of this programme at 12 months of follow-up and to identify potential predictors of a positive outcome, defined as a weight loss $\geq 10 \%$ of initial weight. This target has shown to reduce several obesity-related risk factors (National Institutes of Health, 2000), although in patients with severe obesity this cut-off must be inferred from evidence of the benefits in less obese individuals. Therefore, a weight loss of $10 \%$ at 1 year should be considered as a "minimal goal' in morbidly obese patients.

Furthermore, we tested the hypothesis that high baseline serum folate levels are associated with a more favourable response to the treatment.

\section{Patients and methods}

\section{Study subjects}

We conducted a prospective study of a cohort of 182 morbidly obese patients who started the programme between January 2000 and July 2002. The patients had been sent to the obesity clinic after a first evaluation in the Unit of Endocrinology and Nutrition (Madrid, Spain) if they met the following inclusion criteria: age between 18 and 55 years old, BMI $\geq 40 \mathrm{~kg} / \mathrm{m}^{2}$, high motivation to lose weight and no medical or psychiatric contraindication for bariatric surgery. The patients were evaluated and followed by one endocrinologist (J. J. G.), according to the institutional protocol approved by the hospital.

\section{Protocol}

At baseline, a standard medical history with emphasis on obesity history and associated co-morbidities and a complete physical examination were performed. Weight and height were measured on each individual following the protocol recommended by the Spanish Society for the Study of Obesity (SEEDO) (Aranceta et al. 2003). Dietary intake was assessed by a semiquantitative food history recording weekly consumption of major food groups and the pattern of eating behaviour. At baseline, a fasting blood sample for blood chemistry, thyroid hormones and nutritional tests and a $24 \mathrm{~h}$ urine sample for urinary free cortisol were collected from each subject. Simultaneously, a $75 \mathrm{~g}$ oral glucose tolerance test in non-diabetic patients was performed. Total energy expenditure was estimated by the Harris-Benedict formula using an adjusted body weight ( (actual weight - ideal weight $) \times 0.25+$ ideal weight $)$ and multiplied by an activity factor $(\times 1.2$ if sedentary, $\times 1.4$ if moderately active and $\times 1.6$ if very active). Ideal weight was calculated by the BMI formula assuming an ideal BMI of $22 \mathrm{~kg} / \mathrm{m}^{2}$ for women and $23 \mathrm{~kg} / \mathrm{m}^{2}$ for men. A low-energy diet reducing by $2092 \mathrm{MJ}(500 \mathrm{kcal})$ the estimated total energy expenditure but maintaining a minimal intake of $5021 \mathrm{MJ}(1200 \mathrm{kcal})$ was prescribed. The diet characteristics followed the National Institutes of Health guidelines (National Institutes of Health, 2000) but were adapted to a Mediterranean diet: total fat $\leq 30 \%$ total energy, saturated fatty acids $8-10 \%$ of total energy, MUFA (mainly as olive oil) $\leq 15 \%$ of total energy, PUFA $\leq 10 \%$ of total energy (at least two servings of fish per week), cholesterol $<300 \mathrm{mg} / \mathrm{d}$, protein $15 \%$ of the total energy, carbohydrate $>55 \%$ of total energy, five servings of fruit and vegetables per $\mathrm{d}$ (at least one citrus fruit per $\mathrm{d}$ ), alcohol intake limited to one glass of wine per $\mathrm{d}$ for women and two glasses for men, sodium chloride $<6 \mathrm{~g} / \mathrm{d}$, Ca $1000-1500 \mathrm{mg} / \mathrm{d}$ and fibre $20-30 \mathrm{~g} / \mathrm{d}$. No patient received multivitamin preparations.

Moderate-intensity aerobic exercise (walking, cycling, swimming), 30-45 min/d, 3-5 d/week was recommended.

If a patient failed to lose $5 \%$ of initial weight at 3 months or weight recovery was observed during the follow-up, drug therapy with orlistat or sibutramine was indicated. Orlistat $(120 \mathrm{mg}$ three times per d) was considered as the first-line drug in patients with hypertension, type 2 diabetes mellitus, impaired glucose tolerance, dyslipaemia or concomitant antidepressant therapy. Sibutramine $(10-15 \mathrm{mg}$ once per d) was prescribed in normotensive patients, not taking serotonin reuptake inhibitors, with history of binge eating, complaints of frequent feelings of hunger or poor tolerance to the hypoenergetic diet. In patients with depressed mood or bulimic behaviour, the use of serotonin reuptake inhibitors (fluoxetine $(20-60 \mathrm{mg} / \mathrm{d})$ or sertraline $(50-100 \mathrm{mg} / \mathrm{d}))$ was considered.

Every patient received written materials following the National Institutes of Health guidelines (National Institutes of Health, 2000) in order to modify the eating behaviour. Body weight, blood pressure and treatment adherence were monitored by an endocrinologist and a nurse in individual sessions. Patients' compliance was assessed through repeated records of the semiquantitative food history and physical activity. A lifestyle-change programme according to the National Institutes of Health guidelines was conducted in order to modify eating practices, habits of physical activity and compliance difficulties. This programme included patients' self-monitoring, stimulus control techniques and stress management. An incremental approach to lifestyle change, instead of simultaneous achievement of multiple goals, was recommended. Psychiatric consultation was ordered in patients with mood disorders, bulimia nervosa or binge eating disorder. A weight loss $\geq 5 \%$ of the initial weight at 3 months and $\geq 10 \%$ at 6 months were set as initial goals, recommending a weight-loss rate of $0.5-1 \mathrm{~kg} / \mathrm{week}$. Positive outcome at 12 months was defined as a weight loss of at least $10 \%$ of the initial weight, because after the first 6 months of treatment, the rate at which the weight is lost usually declines and then plateaus.

\section{Assessment of variables}

Potential predictive factors to a programme response were classified into clinical and nutritional variables. Additionally, several follow-up variables were recorded after starting the treatment. 
Clinical variables included age, sex, height, baseline weight and BMI, familial history of obesity, age of onset, response to previous diets, smoking, alcohol intake, energy content of the prescribed diet, servings of fruit and vegetables per week, physical activity, CHD, hypertension (defined by Joint National Commission VII criteria or therapy with antihypertensive drugs), hypercholesterolaemia and hypertriacylglycerolaemia (defined by National Cholesterol Education Program III criteria or treatment with lipid-lowering agents), type 2 diabetes mellitus, impaired fasting glucose or impaired glucose tolerance (defined by American Diebetes Association criteria), hyperuricaemia (defined by uric acid levels $\geq 60 \mathrm{mg} / \mathrm{l}$ in women or $\geq 70 \mathrm{mg} / \mathrm{l}$ in men or use of alopurinol), sleep apnoea (defined by continuous positive airway pressure therapy, diagnostic polysomnography or positive pulse oximetry plus symptoms), primary hypothyroidism (levothyroxine therapy or thyroid-stimulating hormone $>4.5 \mathrm{mU} / \mathrm{l}$ in two samples), osteoarthritis, mood disorders (dysthymic disorder or major depression defined by the Diagnostic and Statistical Manual of Mental Disorders IV criteria) and eating disorders (bulimia nervosa or binge eating disorder by the Diagnostic and Statistical Manual of Mental Disorders IV criteria).

Nutritional variables comprised serum levels of albumin, transferrin, prealbumin, retinol-binding protein, $\mathrm{Zn}$, folic acid, vitamin $\mathrm{B}_{12}, \mathrm{Ca}, \mathrm{P}, \mathrm{Mg}$, parathyroid hormone and 25-hydroxyvitamin $\mathrm{D}_{3}$.

Follow-up variables included number of clinical visits, adherence to diet, smoking status, and number of months with orlistat, sibutramine and serotonin reuptake inhibitors treatment.

\section{Methods}

Plasma glucose and serum levels of lipids, uric acid, albumin, $\mathrm{Ca}, \mathrm{P}$ and $\mathrm{Mg}$ were determined by a colorimetric technique with a Modular Roche DPP analyser (Modular Roche DPP, Hitachi Ltd, Tokyo, Japan). Serum transferrin and ferritin were measured by immunoturbidimetry (Modular Roche DPP). Serum prealbumin and retinol-binding protein were analysed by a nephelometric immunoassay (BN II; Dade Behring Diagnostic, Marburg, Germany). Serum folic acid, vitamin $\mathrm{B}_{12}$ and parathyroid hormone were determined by an electrochemiluminometric assay (Modular Roche E170). Serum free thyroxine and thyroid-stimulating hormone concentrations and urinary free cortisol levels were measured by an immunochemiluminometric assay (Ortho-Clinical Diagnostics, Raritan, NJ, USA). Plasma Zn was measured by atomic-absorption spectroscopy and a RIA was used to measure 25 -hydroxyvitamin $\mathrm{D}_{3}$.

\section{Statistical analysis}

The binary variable 'weight loss $\geq 10 \%$ at 12 months' was the dependent variable. The patients who dropped out of the programme were considered as non-responders. So, the entire cohort was divided into two groups according to the response to the therapy at 12 months of follow-up (group 1, responders; group 2, non-responders or withdrawal). Inclusion of dropouts in the group of non-responders is a conservative methodological assumption, because the most common cause of study discontinuation in weight-loss trials is the lack of effect of therapy. Notwithstanding, an additional analysis of noncompleters to define their features was performed.
Categorical data are presented as percentages and $95 \%$ CI. Continuous variables are shown as means and $95 \%$ CI unless otherwise mentioned. Comparison of categorical variables between both groups was tested by $\chi^{2}$ analysis or the Fisher exact test when appropriate. A linear trend $\chi^{2}$ test was used in the case of variables with ordered categories. Data were tested for normality of distribution by the Shapiro-Wilk test. Comparison of means was performed by Student's $t$ test and a Mann-Whitney U test was used if distribution departed from normality. Associations between continuous variables were tested by Pearson's correlation coefficient or Spearman's rank correlation coefficient in non-normal distributions.

Potential confounders such as sex, age, baseline BMI, tobacco, alcohol and vitamin $\mathrm{B}_{12}$, along with serum folate and those variables with $P<0 \cdot 1$ in responder $v$. non-responder comparison, were included in a binary logistic regression model in order to identify baseline variables for the prediction of a successful outcome in the programme. Linearity in the logit of continuous covariates was tested by the Box-Tidwell transformation. Interaction terms which are the cross-product of each covariate multiplied by its natural logarithm were added to the logistic model. If these terms are significant, then there is non-linearity in the logit and these variables were categorised into quintiles before their inclusion in the model. Adjusted odd ratios $(\mathrm{OR})$ for the confounding and interaction effects of control variables, with their $95 \% \mathrm{CI}$, were estimated following the algorithm proposed by Kleinbaum \& Klein (2002). Interactions between serum folate and other variables of interest were initially tested by the likelihood ratio test, maintaining the significant interactions $(P<0.05)$ in the model. In a second stage, those variables which did not cause a meaningful change in the adjusted OR of the main explanatory variable were removed from the full model. A meaningful change in this OR was defined as a difference $\geq 10 \%$ between the OR of the full model containing all potential confounders and the OR of the reduced model that deletes one or more eligible variables. Statistical significance was assumed at $P<0 \cdot 05$.

Although there are several guidelines which establish a weight-loss goal of $10 \%$ as a successful outcome in the non-surgical treatment of the obese patient, this arbitrary cut-off at 1 year is not based on clear-cut epidemiological results in morbid obesity and might provide artifactual results in the present study. Therefore, we also performed a multiple linear regression using percentage of weight loss as a continuous variable in order to evaluate the effect of serum folate on the outcome after adjusting for potential confounders. Selection criteria of the control variables and backward deletion of confounders were similar to those explained in the binary logistic regression model but in this case adjusted unstandardised B coefficients were estimated.

The SPSS 10.0 statistical package (SPSS Inc., Chicago, IL, USA) was used for the calculations.

\section{Results}

\section{Results of the management programme}

Baseline clinical and laboratory data of the cohort are presented in Tables 1 and 2. Fifty-four patients $(29.7 \%)$ dropped out of the study. The median number of visits was 6 (interquartile range (IQR) 5-7 visits). Median prescribed energy intake was 5439 
(IQR 5021-6276) MJ/d (1300 (IQR 1200-1500) kcal/d). Of the patients, $15.4 \%$ were taking serotonin uptake inhibitors at baseline, and $28 \%$ were treated with fluoxetine or sertraline during the programme (time of treatment 6 (IQR 3-12) months. After 3 months, $40 \%$ of the patients were treated with orlistat (time of therapy 4 (IQR 3-8) months) and 10\% with sibutramine (time of therapy 2 (IQR 2-3) months).

Fig. 1 shows the response of the entire cohort to the programme. In the patients who completed the treatment, the median weight decreased from 114 (IQR 106.0-125.6) $\mathrm{kg}$ at baseline to 108.9 (IQR $98.5-120.3$ ) $\mathrm{kg}$ at 12 months, representing a percentage loss of $4.5 \%$. The BMI decrease was also modest: the median BMI decreased from 44.1 (IQR $41.2-47.9$ ) $\mathrm{kg} / \mathrm{m}^{2}$ at baseline to 42.6 (IQR $38.7-47.4$ ) $\mathrm{kg} / \mathrm{m}^{2}$ at 1 year. At 12 months, twenty-one patients $(11.5 \%$ of the initial cohort, $16.5 \%$ of the completers) had lost $\geq 10 \%$ of their initial weight. In this group the median baseline weight and BMI were 114.3 (IQR 101.6-125.3) $\mathrm{kg}$ and 43.8 (IQR $41.8-45.2) \mathrm{kg} / \mathrm{m}^{2}$, respectively; at 12 months the median weight and BMI were 96 (IQR 87.3-106.5) $\mathrm{kg}$ and 36.9 (IQR $36 \cdot 0-38 \cdot 4) \mathrm{kg} / \mathrm{m}^{2}$, respectively.

\section{Baseline predictors of successful outcome}

When comparing responders to non-responders, only the variables 'serum folate' and 'ferritin' were significantly associated with a positive response at 12 months (Table 2). Low serum $\mathrm{P}$ showed a non-significant trend. In the adjusted model, the interactions folate $\times$ age, folate $\times$ sex, folate $\times$ smoking, folate $\times$ alcohol and folate $\times$ BMI were tested, but no significant interaction was found. The full model included serum folate level as the main explanatory variable and the variables sex, age, baseline BMI, smoking, alcohol, vitamin $\mathrm{B}_{12}$, ferritin and phosphate as potential confounders. The final model retained the variables serum folate, age and smoking. A high serum level of folate at the start of the programme was the only factor significantly associated with a successful outcome (Table 3). Every $1 \mathrm{ng} / \mathrm{ml}$ increment of serum folate increased the chance of success by $28(95 \% \mathrm{CI} 4,58) \%$. After entering the folate categorised in quintiles, the patients with serum folate levels in the top quintile $(>7.2 \mathrm{ng} / \mathrm{ml})$ showed a likelihood of loss $\geq 10 \%$ of initial weight at 1 year, 8.5 times higher than the patients in the two lower quintiles $(<4.7 \mathrm{ng} /$ ml) (Fig. 2 (A)). Both full and final models were statistically significant (full model $-2 \log$ likelihood statistic 58.6 and Nagelkerke $R^{2} 0 \cdot 243, P<0 \cdot 05$; final model $-2 \log$ likelihood statistic 65.0 and Nagelkerke $\left.R^{2} 0.133, P<0.05\right)$.

In a multiple linear regression model with the percentage of weight loss as the outcome variable, baseline serum folate remained as a significant predictor of weight loss after adjusting for the same variables entered in the logistic regression model (Table 4). An increment of $1 \mathrm{ng} / \mathrm{ml}$ in serum folate was associated with an adjusted percentage loss of 0.53 (95\% CI 0.07, 1.0) points (Fig. 2 (B)). No other variables were significant predictors of weight loss at 1 year in this model.

Table 1. Baseline clinical characteristics of study subjects*

(Percentages, mean values and $95 \% \mathrm{Cl}$ )

\begin{tabular}{|c|c|c|c|c|c|c|c|c|c|c|}
\hline \multirow[b]{2}{*}{ Characteristics } & \multirow[b]{2}{*}{ Mean } & \multicolumn{2}{|c|}{ Total (n 182) } & \multirow[b]{2}{*}{ Mean } & \multicolumn{2}{|c|}{$\begin{array}{c}\text { Group } 1 \\
\text { (responders; } n \text { 21) }\end{array}$} & \multirow[b]{2}{*}{ Mean } & \multicolumn{2}{|c|}{$\begin{array}{c}\text { Group } 2 \\
\text { (non-responders; } \\
n 161)\end{array}$} & \multirow[b]{2}{*}{$P$} \\
\hline & & $\%$ & $95 \% \mathrm{Cl}$ & & $\%$ & $95 \% \mathrm{Cl}$ & & $\%$ & $95 \% \mathrm{Cl}$ & \\
\hline Females & & 78 & $71 \cdot 3,83 \cdot 8$ & & $76 \cdot 2$ & $52 \cdot 8,91 \cdot 8$ & & $78 \cdot 3$ & $71 \cdot 1,84 \cdot 4$ & 0.784 \\
\hline Age (years) & $40 \cdot 5$ & & $38 \cdot 8,42 \cdot 2$ & $40 \cdot 7$ & & $38 \cdot 9,42 \cdot 5$ & 39 & & $33 \cdot 7,44 \cdot 2$ & 0.53 \\
\hline Weight (kg) & $116 \cdot 4$ & & $114 \cdot 0,118 \cdot 8$ & 114.5 & & $107 \cdot 9,121 \cdot 2$ & $116 \cdot 6$ & & $114 \cdot 1,119 \cdot 2$ & 0.573 \\
\hline BMI $\left(\mathrm{kg} / \mathrm{m}^{2}\right)$ & $45 \cdot 4$ & & $44 \cdot 5,46 \cdot 3$ & $45 \cdot 0$ & & $42 \cdot 1,47 \cdot 8$ & $45 \cdot 4$ & & $44 \cdot 5,46 \cdot 4$ & 0.658 \\
\hline Family history of severe obesity & & $9 \cdot 8$ & $5 \cdot 8,15 \cdot 2$ & & $5 \cdot 0$ & $0.1,24.9$ & & $10 \cdot 4$ & $6 \cdot 1,16 \cdot 3$ & 0.697 \\
\hline Age of onset of obesity & & & & & & & & & & 0.264 \\
\hline$<10$ years & & 41.5 & $34 \cdot 0,49 \cdot 3$ & & 50 & $27 \cdot 2,72 \cdot 8$ & & $40 \cdot 4$ & $32 \cdot 5,48 \cdot 7$ & \\
\hline $10-18$ years & & $21 \cdot 1$ & $15 \cdot 2,27 \cdot 9$ & & 25 & $8 \cdot 7,49 \cdot 1$ & & 20.5 & $14 \cdot 4,27 \cdot 9$ & \\
\hline$>18$ years & & $37 \cdot 4$ & $30 \cdot 2,45 \cdot 1$ & & 25 & $8 \cdot 7,49 \cdot 1$ & & $39 \cdot 1$ & $31 \cdot 2,47 \cdot 3$ & \\
\hline Good previous response to diets & & $52 \cdot 8$ & $45 \cdot 2,60 \cdot 2$ & & 61.9 & $38 \cdot 4,81 \cdot 9$ & & $51 \cdot 6$ & $43 \cdot 5,59 \cdot 6$ & 0.549 \\
\hline Bulimia or binge-eating disorder & & 11 & $6 \cdot 9,16 \cdot 5$ & & $9 \cdot 5$ & $1 \cdot 2,30 \cdot 4$ & & $11 \cdot 3$ & $6 \cdot 8,17 \cdot 2$ & 1 \\
\hline Smokers & & $29 \cdot 7$ & $23 \cdot 1,36 \cdot 9$ & & 42.9 & $21 \cdot 8,66$ & & 28 & $21 \cdot 2,35 \cdot 6$ & $0 \cdot 16$ \\
\hline Type 2 diabetes mellitus & & $21 \cdot 4$ & $15 \cdot 7,28 \cdot 1$ & & 19 & $5 \cdot 4,41 \cdot 9$ & & $21 \cdot 7$ & $15 \cdot 6,28 \cdot 9$ & 1 \\
\hline Prediabetes (IGT or IFG) & & $15 \cdot 3$ & $10 \cdot 5,21 \cdot 5$ & & $4 \cdot 8$ & $0 \cdot 1,23 \cdot 8$ & & $16 \cdot 8$ & $11 \cdot 4,23 \cdot 5$ & 0.207 \\
\hline Hypretension & & $46 \cdot 7$ & $39 \cdot 3,54 \cdot 2$ & & 61.9 & $38 \cdot 4,81 \cdot 9$ & & $44 \cdot 7$ & $36 \cdot 9,52 \cdot 7$ & 0.138 \\
\hline Hypercholesterolaemia & & $37 \cdot 4$ & $30 \cdot 3,44 \cdot 8$ & & $28 \cdot 6$ & $11 \cdot 3,52 \cdot 2$ & & 38.5 & $31,46 \cdot 5$ & 0.376 \\
\hline Hypertriacylglycerolaemia & & $14 \cdot 3$ & $9 \cdot 5,20 \cdot 2$ & & $9 \cdot 5$ & $11 \cdot 7,30 \cdot 4$ & & $14 \cdot 9$ & $9 \cdot 8,21 \cdot 4$ & 0.743 \\
\hline Hyperuricaemia & & $17 \cdot 1$ & $11.9,23.4$ & & 10 & $1 \cdot 2,31 \cdot 7$ & & 18 & $12 \cdot 4,24 \cdot 8$ & 0.534 \\
\hline Sedentary lifestyle & & $74 \cdot 6$ & $67 \cdot 6,80 \cdot 8$ & & 71.4 & $47 \cdot 8,88 \cdot 7$ & & 75 & $67 \cdot 6,81 \cdot 5$ & 0.724 \\
\hline $\mathrm{CHD}$ & & $2 \cdot 2$ & $0.6,5.5$ & & $4 \cdot 8$ & $0 \cdot 1,23 \cdot 8$ & & 1.9 & $0.4,5.3$ & 0.39 \\
\hline Sleep apnoea & & $22 \cdot 1$ & $16 \cdot 3,28 \cdot 9$ & & $14 \cdot 3$ & $3 \cdot 0,36 \cdot 3$ & & $23 \cdot 2$ & $16 \cdot 8,30 \cdot 4$ & 0.575 \\
\hline Primary hypothyroidism & & 22 & $16 \cdot 2,28 \cdot 7$ & & $14 \cdot 3$ & $3 \cdot 0,36 \cdot 3$ & & 23 & $16 \cdot 7,30 \cdot 3$ & 0.575 \\
\hline Osteoarthritis & & 31.9 & $25 \cdot 3,39 \cdot 4$ & & $28 \cdot 6$ & $11 \cdot 3,52 \cdot 2$ & & 32.5 & $25 \cdot 3,40 \cdot 4$ & 0.717 \\
\hline Mood disorder & & 32 & $25 \cdot 3,39 \cdot 4$ & & 19 & $5.4,41.9$ & & $33 \cdot 8$ & $26 \cdot 5,41 \cdot 6$ & 0.175 \\
\hline Serotonin uptake inhibitor therapy & & $15 \cdot 4$ & $10 \cdot 5,21 \cdot 5$ & & $9 \cdot 5$ & $1 \cdot 2,30 \cdot 4$ & & $16 \cdot 1$ & $10 \cdot 8,22 \cdot 8$ & 0.747 \\
\hline
\end{tabular}

IGT, impaired glucose tolerance; IFG, impaired fasting glucose.

*Data from the entire cohort and from the two subgroups classified according to the outcome at 1 year are shown. The statistical significance of differences between both groups (responders and non-responders) is shown. 


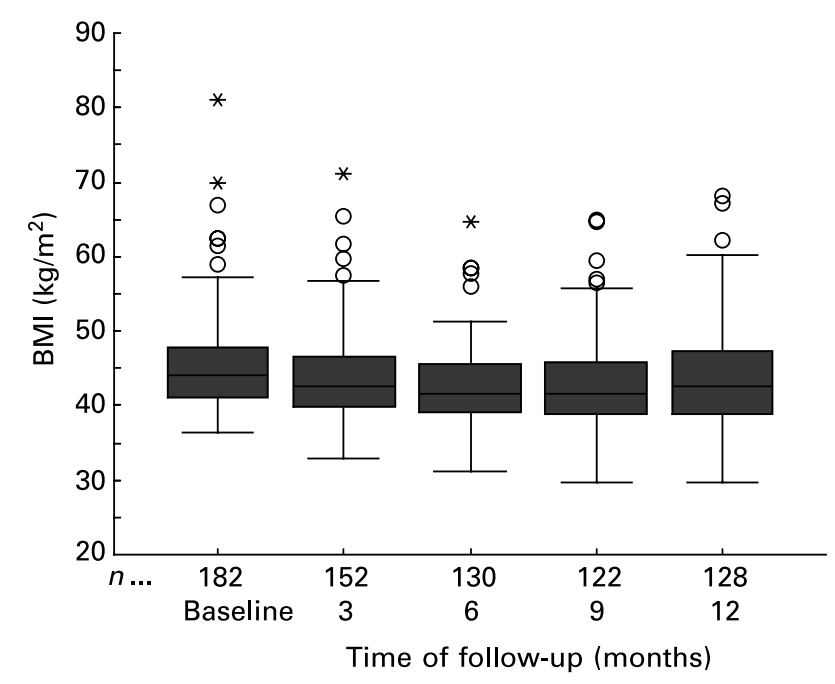

Fig. 1. BMI change in the patients of the study from enrolment until 12 months of follow-up. Data are represented as box and whisker plots. (-), Median BMI. Each box represents the interquartile range (IQR) containing $50 \%$ of the values. Whiskers extend to the upper and lower adjacent values that are 1.5 times or less the IQR. (O), Outliers (values between 1.5 and 3 times the IQR) (individually plotted); ( *) extremes (values more than 3 times the IQR).

In a secondary analysis, a positive correlation between serum folic acid and weekly intake of fruit and vegetables was observed (Spearman's $\rho 0.4 ; P<0.001$ ) (Fig. 3). Only $11.4 \%$ of patients consumed $\geq$ five servings of fruit plus vegetables at baseline. The intake of fruit and vegetables was not a significant predictor after its inclusion in the former multiple linear regression model.

There were no significant differences in the recommended energy intake between both groups $(P=0 \cdot 84)$. The responders showed a better adherence to the programme; $76.2 \%$ of these patients had a good compliance with dietary recommendations $v .33 .8 \%$ of non-responders $(P<0.001)$. Median number of visits was six in the successful subgroup $v$. five in non-responders $(P=0 \cdot 09)$. Six of fifty-four smokers stopped smoking during the study (one responder and five non-responders $(P=0 \cdot 795)$.

Treatment with orlistat, sibutramine or serotonin reuptake inhibitors was not associated with a weight loss $\geq 10 \%$ at 12 months. Of orlistat-treated patients, $13.7 \%$ lost $\geq 10 \%$ initial weight $v .10 \cdot 1 \%$ of non-treated patients $(P=0.455)$. Of sibutramine-treated patients, $5.6 \%$ reached the weight goal v. $12.3 \%$ of non-treated patients $(P=0 \cdot 7)$. Finally, $9.8 \%$ of patients taking serotonin reuptake inhibitors were responders $v .12 .2 \%$ of non-treated patients $(P=0.65)$. Every multivariate analysis was repeated entering these follow-up variables as potential confounders but all of them were deleted from the final models.

A significant percentage of the cohort showed mood disorders $(32 \%)$ or eating disorders $(11 \%)$. No statistically significant differences in the prevalence of these pathologies were observed between responders and non-responders, although more patients in the group of non-responders were diagnosed with mood disorders (33.8 v. $19 \% ; P=0.175)$. We evaluated the serum folate levels and the intake of fruit and vegetables stratified by both subgroups of patients and no significant differences were found. Mean serum folate 
Table 3. Adjusted odds ratios (OR) and statistical significance of likelihood ratio test for the association between potential predictors and a weight loss $\geq 10 \%$ of the baseline weight at 1 year*

(Adjusted odds ratios and $95 \% \mathrm{Cl}$ )

\begin{tabular}{|c|c|c|c|c|c|c|}
\hline \multirow[b]{2}{*}{ Variables } & \multicolumn{3}{|c|}{ Full model } & \multicolumn{3}{|c|}{ Final model } \\
\hline & OR & $95 \% \mathrm{Cl}$ & $P$ & OR & $95 \% \mathrm{Cl}$ & $P$ \\
\hline Folate (×1 ng/ml increment) & $1 \cdot 31$ & $1.03,1.67$ & 0.029 & $1 \cdot 28$ & $1.04,1.58$ & 0.026 \\
\hline $\mathrm{BMI}\left(\times 1 \mathrm{~kg} / \mathrm{m}^{2}\right)$ & 1.02 & $0.92,1.14$ & 0.803 & & & \\
\hline Age ( $\times 1$ year increment $)$ & 0.95 & $0.88,1.02$ & $0 \cdot 154$ & & & \\
\hline Sex (female $v$. male) & 1.59 & $0 \cdot 11,22 \cdot 35$ & 0.730 & & & \\
\hline Smoker (yes $v$. no) & 0.30 & $0.07,1.27$ & $0 \cdot 102$ & 0.26 & $0.06,1.03$ & 0.055 \\
\hline Alcohol (moderate intake $v$. no intake) & 1.07 & $0.08,14.6$ & 0.958 & & & \\
\hline Ferritin $(\times 1 \mathrm{ng} / \mathrm{ml}$ increment $)$ & 1.01 & $0.99,1.01$ & $0 \cdot 151$ & & & \\
\hline $\mathrm{P}(\times 1 \mathrm{ng} / \mathrm{ml}$ increment $)$ & 0.53 & $0 \cdot 16,1 \cdot 77$ & 0.300 & & & \\
\hline Vitamin $B_{12}(\times 1 \mathrm{pg} / \mathrm{ml}$ increment $)$ & 1.00 & $0.99,1.01$ & 0.942 & & & \\
\hline
\end{tabular}

*Initial full model and final reduced model (after removing those variables that do not cause a change in the OR of the folate $\geq 10 \%)$ are displayed. In both models high serum folate levels showed a significant association with a positive outcome.

Table 4. Adjusted unstandardised B coefficients and statistical significance of $t$ test for the association between potential predictors and the percentage of weight loss $\geq 10 \%$ at 1 year*

(B coefficients and $95 \% \mathrm{Cl}$ )

\begin{tabular}{|c|c|c|c|c|c|c|}
\hline \multirow[b]{2}{*}{ Variables } & \multicolumn{3}{|c|}{ Full model } & \multicolumn{3}{|c|}{ Final model } \\
\hline & $\mathrm{B}$ & $95 \% \mathrm{Cl}$ & $P$ & $\mathrm{~B}$ & $95 \% \mathrm{Cl}$ & $P$ \\
\hline Folate (×1 ng/ml increment) & 0.57 & $0.04,1.09$ & 0.035 & 0.53 & $0.07,1.0$ & 0.024 \\
\hline $\mathrm{BMI}\left(\times 1 \mathrm{~kg} / \mathrm{m}^{2}\right)$ & $-1 \cdot 12$ & $-0.36,0.13$ & 0.348 & & & \\
\hline Age ( $\times 1$ year increment $)$ & -0.05 & $-0.17,0.08$ & 0.439 & & & \\
\hline Sex (female $v$. male) & 0.01 & $-4 \cdot 69,4 \cdot 70$ & 0.997 & & & \\
\hline Smoker (yes $v$. no) & 1.39 & $-1.55,4.33$ & 0.348 & & & \\
\hline Alcohol (moderate intake $v$. no intake) & $-3 \cdot 31$ & $-8 \cdot 39,1 \cdot 77$ & 0.198 & & & \\
\hline Ferritin ( $\times 1 \mathrm{ng} / \mathrm{ml}$ increment) & 0.01 & $-0.008,0.03$ & 0.29 & 0.01 & $-0.002,0.02$ & 0.099 \\
\hline $\mathrm{P}(\times 1 \mathrm{ng} / \mathrm{ml}$ increment $)$ & -0.30 & $-2 \cdot 40,1 \cdot 80$ & 0.778 & & & \\
\hline Vitamin $\mathrm{B}_{12}(\times 1 \mathrm{pg} / \mathrm{ml}$ increment $)$ & 0.003 & $-0.004,0.009$ & 0.408 & & & \\
\hline
\end{tabular}

*Initial full model and final reduced model (after removing those variables that do not cause a change in the B coefficient of the folate $\geq 10 \%$ ) are shown. In both models baseline serum folate remained as a significant predictor of weight loss.

was $5.7 \mathrm{ng} / \mathrm{ml}$ in patients with mood disorders $(v .5 .6 \mathrm{ng} / \mathrm{ml}$ in the rest of the cohort; $P=0.64)$ and $4.9 \mathrm{ng} / \mathrm{ml}$ in patients with eating disorders $(v .5 .7 \mathrm{ng} / \mathrm{ml}$ in the rest of the patients; $P=0 \cdot 41$ ). Mean weekly intake of fruit and vegetables was eighteen servings in the group with mood disorders or eating disorders $v$. nineteen servings in the rest of the patients $(P=0.42$ and 0.45 respectively).

\section{Analysis of patients with loss of follow-up}

We performed an additional study to define the features of the patients who dropped out of the programme. When comparing dropouts with non-dropouts, being male was associated with more withdrawals $(42.5 \%$ in men v. $26.1 \%$ in women; $P=0.044$ ), while a diagnosis of type 2 diabetes mellitus $(12.8 \%$ in diabetics $v .34 .3 \%$ in non-diabetic patients; $P=0.009)$ or primary hypothyroidism $(15 \%$ in hypothyroid v. $33.8 \%$ in euthyroid patients; $P=0.021)$ were associated with a higher percentage of completion. These differences, however, turned non-significant in a binary logistic regression model entering the variable 'withdrawal' as the response variable. Most non-completer participants who could be contacted had dropped out because of a poor response to the treatment or a lack of adherence to the hypoenergetic diet. No deaths were recorded.

\section{Discussion}

In the present study, a medical-management programme of morbid obesity obtained limited results at 1 year. The median percentage of weight loss at 12 months was $4.5 \%$ and only $11.5 \%$ of the patients who started the programme had a successful outcome, losing at least $10 \%$ of the initial weight. Furthermore, a high proportion of withdrawals was observed $(29.7 \%)$. Even if the analysis is performed excluding the missing cases, the present results remain poor, because only $16 \%$ of the patients who completed 1 year of followup achieved the minimal goal of weight loss.

The limited long-term success of non-surgical approaches in obesity has been frequently reported. Several studies show that medical interventions comprising nutritional therapy adjunct to lifestyle changes, physical exercise, behavioural therapy and drug therapy with orlistat or sibutramine, obtain a weight loss about $10 \%$ in the short to medium term (Miller et al. 1997; Padwal et al. 2003). Nevertheless, in the long term these therapies usually have discouraging results, since 

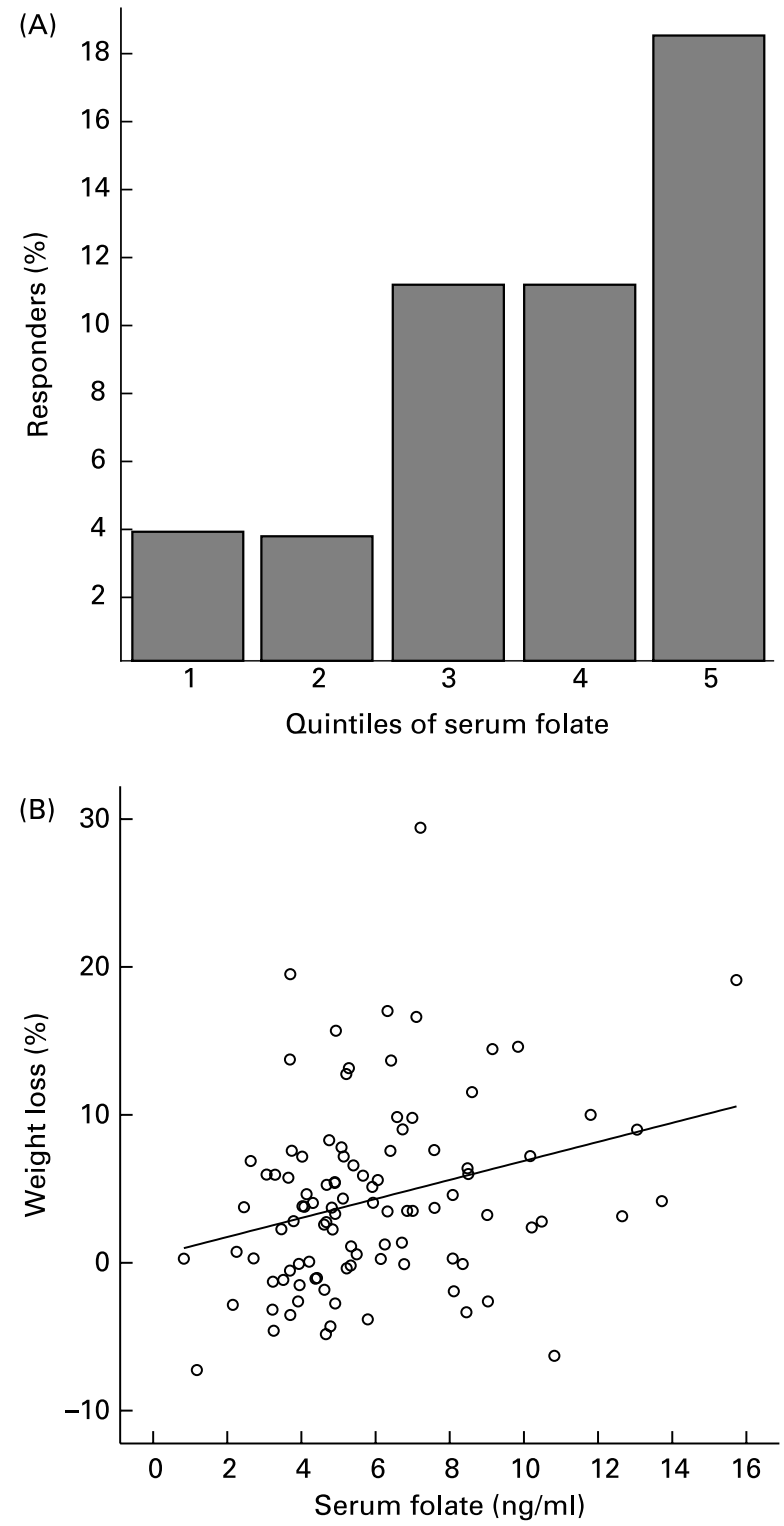

Fig. 2. (A) Baseline serum folate levels (categorised into quintiles) and likelihood of a weight loss $\geq 10 \%$ of initial weight at 12 months. Of patients with serum folate levels within the top quintile, $19 \%$ achieved the objective compared with $<4 \%$ of patients with folate concentrations within the two lower quintiles (odds ratio 8.5 adjusted for potential confounders). (B) Baseline serum folate levels and percentage of weight loss at 12 months. A positive correlation between folate and weight loss was observed. An increment of $1 \mathrm{ng} / \mathrm{ml}$ in serum folate was associated with an adjusted weight loss of 0.53 $(95 \% \mathrm{Cl} 0.07,1.0) \%$.

most patients regain their lost weight within 5 years (Wadden, 1993). A systematic review of studies evaluating dietary treatment of obesity and published between 1931 and 1999 pointed to an overall median long-term success rate (maintenance of the weight initially lost at least for 3 years) of $15 \%$ (Ayyad \& Andersen, 2000). In a systematic review and meta-analysis of randomised controlled studies of approved anti-obesity medications with follow-up periods of 1 year or greater showed that, compared with placebo, orlistat-treated patients displayed a $2.7(95 \%$ CI $2.3,3.1) \mathrm{kg}$ or $2.9(95 \%$ CI $2.3,3.4) \%$ greater reduction in weight and patients on sibutramine displayed a $4.3(95 \%$ CI $3.6,4.9) \mathrm{kg}$ or 4.6

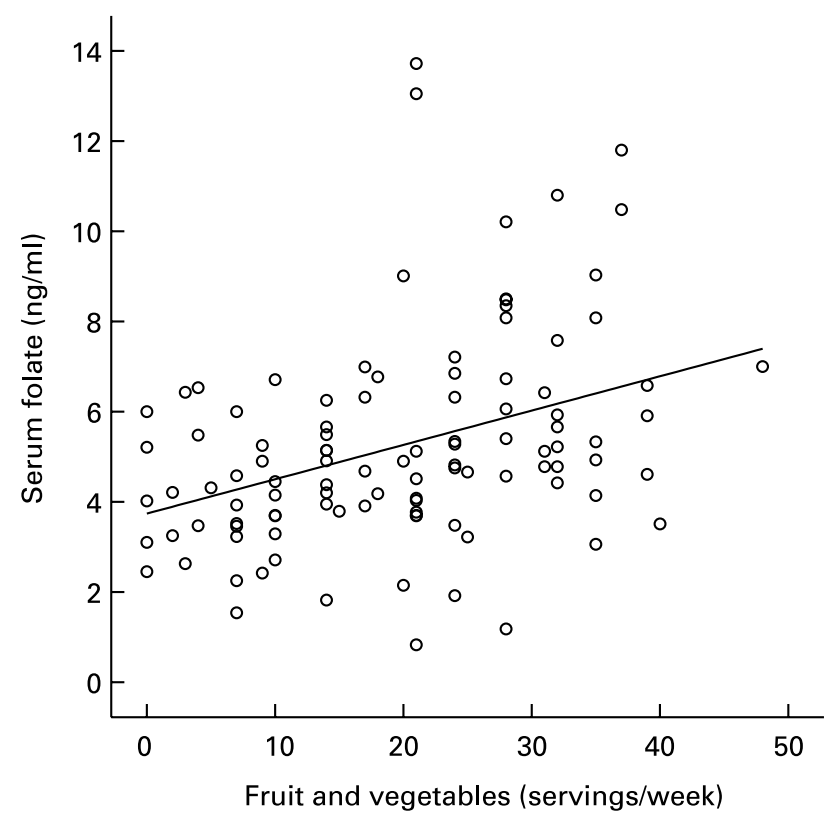

Fig. 3. Correlation between weekly intake of fruit and vegetable servings and serum folate levels at baseline (Spearman's $\rho 0.4$ ).

$(95 \%$ CI 3.8, 5.4) \% greater weight reduction after 1 year of follow-up (Padwal et al. 2003). In the present study, we did not get better results in the pharmacologically treated patients. These findings must be interpreted with caution, because antiobesity drugs were not randomly prescribed at baseline, but after the third month of follow-up according to the institutional protocol, and they were probably indicated in a selected subgroup of non-responder patients.

Theoretically, we should expect lifestyle or pharmacological interventions to obtain poor results in patients with morbid obesity. Before the report of the Swedish Obese Subjects study, the potential efficacy of these approaches in morbid obesity had to be inferred from evidence of their efficacy in less obese individuals. The Swedish Obese Subjects study recruited 1879 severely obese patient pairs in order to compare surgical $v$. conventional obesity treatment (Sjöström et al. 2004). The conventional treatment was not standardised and ranged from sophisticated lifestyle intervention and behaviour modification to, in some practices, no treatment whatsoever. After 2 years, the weight had increased by $0.1 \%$ in the control group and had decreased by $23.4 \%$ in the surgery group. After 10 years, the weight had increased by $1.6 \%$ and decreased by $16.1 \%$, respectively. The present report confirms the limited results of the non-surgical options in morbid obesity.

Therefore, at the present time, the only therapeutic approach able to improve the results of medical treatment in morbid obesity is bariatric surgery (Clegg et al. 2003; Sjöström et al. 2004). In 1991, the National Institutes of Health Consensus Development Conference on Gastrointestinal Surgery for Severe Obesity set that those patients judged by experienced clinicians to have a low probability of success with non-surgical measures might be considered for surgery (National Institutes of Health Consensus Development Conference Statement; Anonymous, 1992). However, it was recognised that there were insufficient data on which to base 
recommendations for patient selection using objective clinical features alone.

The reason why most morbidly obese patients do not succeed at losing weight and sustaining changes in eating behaviour remains largely unknown. Previous non-successful weight-loss attempts and high attrition rates (47.2 and $29.7 \%$ respectively in the present study) are frequently observed in severely obese patients undergoing weight-management programmes. The adherence to the programme was quite poor in our cohort. Only $37.4 \%$ of the patients showed a good compliance with dietary recommendations. Significant differences in the compliance between responders and nonresponders $(76.2$ v. $33.8 \%)$ and a trend to a better completion of the scheduled visits in the first subgroup were observed. Notwithstanding, it is important to recognise that some obese patients are more apt to lose weight on a given programme; this phenomenon cannot always be attributed to the degree of compliance (National Institutes of Health, 2000). Personal factors (genetic, biological, psychological, behavioural) should play a role in determining low success rates, along with treatment features, environmental circumstances and potential interactions among the three (Teixeira et al. 2004). A critical question to consider is whether the patient's level of motivation is high enough to continue the treatment.

In the present report we evaluated the baseline clinical and laboratory features of the patients which allow the prediction of a successful outcome to a non-surgical treatment. The primary objective was to avoid a delay in bariatric surgery in non-responder patients to a 1-year medical programme and to reinforce the therapy in the responders. Serum folate was a good candidate because this is an easily measurable, inexpensive parameter. It had shown in the Health Professionals Follow-up Study a positive correlation with a healthy diet pattern characterised by higher intakes of fruit, vegetables, whole grains and poultry and, conversely, a negative correlation with a Western diet characterised by higher intakes of red meats, high-fat dairy products and refined grains (Fung et al. 2001). After a binary logistic regression analysis, serum folate level was a significant biochemical predictor of success in the present study. In turn, we failed to identify other established prognostic markers of weight-loss success in obesity, such as initial body weight, BMI or previous physical activity (Hansen et al. 2001; Reinehr et al. 2003). These results preclude the definition of a clear-cut 'success clinical profile' in the medical management of morbid obesity.

The patients with high serum levels of folate at the beginning of the programme (top quintile) showed odds of positive outcome about 8.5 times higher than those with low levels (two lower quintiles). A total of $19 \%$ of patients with serum folate $>7.2 \mathrm{ng} / \mathrm{ml}$ lost $>10 \%$ of initial weight compared with $4 \%$ of patients with folate $<4.7 \mathrm{ng} / \mathrm{ml}$ (Fig. 2 (A)). Major sources of folate are orange and other citrus fruits, vegetables, dried fruits, eggs and liver. The serum folate level is quite sensitive to changes in dietary folate intake and is a measure of the status at the time of assay (Herbert, 1999). In the present study, the patients did not receive multivitamin preparations, their serum folate level reflecting the intake of foods rich in folate. Fruit and vegetable intake was very low in the whole group. Only $11.4 \%$ of the patients reported an intake of at least five servings per $\mathrm{d}$ and $24.2 \%$ reported $0-1$ servings per $\mathrm{d}$, but a positive correlation between folate and weekly intake of fruit and vegetables was found. Therefore, folate seems to be a marker of a healthy diet and a surrogate predictor of significant weight loss at 1 year in obese patients undergoing a hypoenergetic diet. Interestingly, serum folate was a better predictor of success than fruit and vegetable intake. In fact, this last variable was not a significant independent predictor after its inclusion in a multiple linear regression model. A semiquantitative food history recording weekly consumption of major food groups suffers from some inaccuracy because is based on the patient's ability to recall, while serum folate measurements provide more reproducible data. On the other hand, high folate might indicate not only a correct intake of fruit and vegetables but also a healthier eating behaviour.

These findings should be regarded with some caution. The validity of the present results needs to be confirmed in other studies, because there are no previous reports evaluating folate as a prognostic marker in obesity. The assay for serum folate reflects only a recent dietary intake and may not reflect tissue stores. Although serum and erythrocyte folate levels decline in parallel, the erythrocyte folate level is a more accurate reflection of tissue stores and serum folate is more sensitive to early negative nutrient balance (1-3 weeks). However, the erythrocyte contains monoglutamate and polyglutamate forms of folic acid, so the doseresponse curves are altered in binding assays. Most studies of folate status in obesity have been performed measuring serum folate concentrations (Hirsch et al. 2005). It would be interesting to study if erythrocyte folate shows similar prognostic value in weight-loss programmes. Unfortunately, the erythrocyte folate assay was not available in our hospital at the beginning of the study.

The large number of statistical comparisons may have increased the chance of type 1 errors. However, the adjusting for multiple potential confounders in the logistic and linear regression analyses suggests that chance alone was not responsible for the reported findings. The conclusions must only be generalised to morbidly obese patients fulfilling surgical criteria. A meaningful percentage of the cohort was constituted by patients affected by eating or mood disorders; this heterogeneity reflects a real-life clinical setting in a morbid-obesity-management programme. Nevertheless, the results can be generalised to these subgroups because their baseline serum folate and their intake of fruit and vegetables were not different from those found in the rest of the cohort. Behavioural or genetic predictors (Bernier \& Avard, 1986; Traverso et al. 2000; Teixeira et al. 2004; Moreno-Aliaga et al. 2005) have not been included in the present study, because the main objective was the identification of simple biochemical or clinical predictive factors. Finally, one-third of the patients of the present study did not complete the programme. High attrition rates are the rule in interventional studies in obesity, because of an unsuccessful response in most cases. So, $33 \%$ of the patients in orlistat studies and $43 \%$ of the patients in sibutramine studies dropped out (Padwal et al. 2003) and 26.4\% of the patients assigned to the control group in the Swedish Obese Subjects study were lost to follow-up (Sjöström et al. 2004). In the present study, although being female and having some chronic pathologies such as type 2 diabetes mellitus or primary hypothyroidism were associated with a less likelihood of withdrawal, 
after adjusting for the control variables in a logistic regression model no significant predictors of dropout were identified. Moreover, a new analysis removing missing cases obtained similar results to those found with the entire cohort.

In summary, medical management of morbid obesity shows rather limited results. It is difficult to find consistent predictors of success. The measurement of baseline serum folate levels could be used as a biomarker of healthy nutritional habits and as a predictor of a successful response to the treatment. A final question that remains to be explored is if an early promotion of changes in nutritional habits in morbidly obese patients with low serum folate levels will improve the poor results of the non-surgical therapies.

\section{References}

Anonymous (1992) Gastrointestinal surgery for severe obesity: NIH Consensus Development Conference Statement. Am J Clin Nutr 55, 615S-619S

Aranceta J, Pérez C, Serra L, et al. (2003) Prevalence of obesity in Spain: results of the SEEDO 2000 study (article in Spanish). Med Clin (Barc) 120, 608-612.

Ayyad C \& Andersen T (2000) Long-term efficacy of dietary treatment of obesity: a systematic review of studies published between 1931 and 1999. Obes Rev 1, 113-119.

Bernier M \& Avard J (1986) Self-efficacy, outcome and attrition in a weight-reduction program. Cogn Ther Res 10, 319-338.

Clegg A, Colquitt J, Sidhu M, Royle P \& Walter A (2003) Clinical and cost effectiveness of surgery for morbid obesity: a systematic review and economic evaluation. Int J Obes 27, 1167-1177.

Drenick EJ, Bale GS, Seltzer FS \& Johnson DG (1980) Excessive mortality and causes of death in morbidly obese men. JAMA 243, 443-445.

Flegal KM, Carroll MD, Ogden CL \& Johnson CL (2002) Prevalence and trends in obesity among US adults, 1999-2000. JAMA 288, $1723-1727$.

Fung TT, Rimm EB, Spiegelman D, Rifai N, Tofler GH, Willett WC \& Hu FB (2001) Association between dietary patterns and plasma biomarkers of obesity and cardiovascular disease risk. Am J Clin Nutr 73, 61-67.

Hansen D, Astrup A, Toubro S, et al. (2001) Predictors of weight loss and maintenance during 2 years of treatment by sibutramine in obesity. Results from the European multi-centre STORM trial. Sibutramine Trial of Obesity Reduction and Maintenance. Int $J$ Obes 25, 496-501.
Herbert V (1999) Folic acid. In Modern Nutrition in Health and Disease, pp. 433-446 [ME Shils, JA Olson, M Shike and AC Ross, editors]. Baltimore, MA: Williams and Wilkins.

Hirsch S, Poniachick J, Avendaño M, Csendes A, Burdiles P, Smok G, Diaz JC \& de la Maza MP (2005) Serum folate and homocysteine levels in obese females with non-alcoholic fatty liver. Nutrition $\mathbf{2 1}$, $137-141$.

Kleinbaum DG \& Klein M (2002) Modeling strategy for assessing interaction and confounding. In Logistic Regression: a Self-learning Text, pp. 191-226 [DG Kleinbaum and M Klein, editors]. New York: Springer-Verlag.

MacDonald KG (2003) Overview of the epidemiology of obesity and the early history of procedures to remedy morbid obesity. Arch Surg 138, 357-360.

Miller WC, Koceja DM \& Hamilton EJ (1997) A meta-analysis of the past 25 years of weight loss research using diet, exercise or diet plus exercise intervention. Int J Obes 21, 941-947.

Moreno-Aliaga MJ, Santos JL, Martí A \& Martínez JA (2005) Does weight loss prognosis depend on genetic make-up? Obes Rev 6, $155-168$.

National Institutes of Health (2000) National Heart, Lung and Blood Institute Obesity Education Initiative Expert Panel on the Identification, Evaluation and Treatment of Overweight and Obesity in Adults. NIH Publication no. 00-4084. www.nhlbi.nih.org

Padwal R, Li SK \& Lau DCW (2003) Long-term pharmacotherapy for overweight and obesity: a systematic review and meta-analysis of randomized controlled trials. Int J Obes 27, 1437-1446.

Reinehr T, Brylak K, Alexy U, Kersting M \& Andler W (2003) Predictors to success in outpatient training in obese children and adolescents. Int J Obes 27, 1087-1092.

Rissanen A, Lean M, Rössner S, Segal KR \& Sjöstrom L (2003) Predictive value of early weight loss in obesity management with orlistat: an evidence-based assessment of prescribing guidelines. Int $J$ Obes 27, 103-109.

Sjöström L, Lindroos AK, Peltonen M, et al. (2004) Lifestyle, diabetes, and cardiovascular risk factors 10 years after bariatric surgery. $N$ Engl J Med 351, 2683-2693.

Teixeira PJ, Going SB, Houtkooper LB, Cussler EC, Metcalfe LL, Blew RM, Sardinha LB \& Lohman TG (2004) Pretreatment predictors of attrition and successful wight management in women. Int $J$ Obes 28, 1124-1133.

Traverso A, Ravera G, Lagattolla V, Testa S \& Adami GF (2000) Weight loss after dieting with behavioral modification for obesity: the predicting efficiency of some psychometric data. Eat Weight Disord 5, 102-107.

Wadden TA (1993) Treatment of obesity by moderate and severe caloric restriction. Results of clinical research trials. Ann Intern Med 1197, 688-693. 